\title{
Study on Shale's Dynamic Damage Constitutive Model Based on Statistical Distribution
}

\author{
Jianjun Liu, ${ }^{1,2}$ Yuan Li, ${ }^{2}$ and Huijuan Zhang ${ }^{2}$ \\ ${ }^{1}$ State Key Laboratory of Oil and Gas Reservoir Geology and Exploitation, Southwest Petroleum University, Chengdu 610500, China \\ ${ }^{2}$ School of Geoscience and Technology, Southwest Petroleum University, Chengdu 610500, China
}

Correspondence should be addressed to Jianjun Liu; liujj0906@sina.com

Received 11 January 2015; Accepted 2 April 2015

Academic Editor: Mohammad Elahinia

Copyright (C) 2015 Jianjun Liu et al. This is an open access article distributed under the Creative Commons Attribution License, which permits unrestricted use, distribution, and reproduction in any medium, provided the original work is properly cited.

The dynamic constitutive model of shale is basic for shale gas reservoir reforming. In order to investigate the dynamic mechanism of shale, a new dynamic damage constitutive model of shale under uniaxial impact load was established based on the statistical damage theory and the laboratory test results of deformation and the damage characteristics under the action of SHPB impact. Compared with the theoretical results, the model can describe shale's mechanical attributes and reveal the fracture damage mechanism as well. The results will provide theoretical basis for hydraulic fracturing on shale and other dynamic reforming technics.

\section{Introduction}

Shale gas is an important unconventional gas resource. Because of the characteristics of low porosity and low permeability, shale gas reservoir must be reformed using hydraulic fracturing or other technics. In order to reveal the law of shale's mechanical property and fracture damage mechanism, a reasonable dynamic constitutive model is necessary for effective fracturing design needs. But few works are directly related to this content. In recent years, due to the increasing exploitation of shale gas, the study of shale's mechanical behavior has become the hot issue in the academic fields and engineering areas. On one hand, the research results can provide basic theoretical foundation for reservoir reforming; on the other hand, they can also provide experimental reference and basic parameters for numerical simulation of hydraulic fracturing.

There are many works that have been done on the dynamic constitutive model of rock. For example, Lindholm et al. (1974) proposed an overstress model [1], but the physical parameters of the model are not clear. Sigenori et al. [2] (1977) and Yu [3] further developed the overstress model and obtained the correction of the overstress model, which can describe the dynamic mechanical behavior of rock material very well; Krajcinovic and Silva [4] and Dougill et al. [5] applied damage mechanics in rock and concrete, and the continuous damage process of rock and concrete was analyzed; in addition, the damage constitutive model is established by using the concept of fracture surface. Chen and Feng [6], Wenxue et al. [7], and Shang and Ning [8] established the constitutive relations equation of rock materials by the Lagrange analysis of experimental results under the blasting load. Some results have been achieved on dynamic constitutive model of rock, but the established constitutive model was mostly only for the ordinary rocks whose research backgrounds are engineering blasting, earthquake, and so on and rarely for shale whose mechanic characteristics are more complex than conventional rock in the research background of hydraulic fracturing.

In this paper, the dynamic damage constitutive model of shale under loading is established based on the theory of damage mechanics. At the same time, by considering the relationship between the mechanics parameters and the strain rate, the correction of the model has been put forward. Moreover, compared with the experimental results, the constitutive model is proved to be reasonable.

\section{Establishment of Dynamic Damage Constitutive Model of Shale}

The large amount of defects within shale makes the quantity of damaged parts increase and the area expand when the shale is under a loading situation, and the increased damage causes 


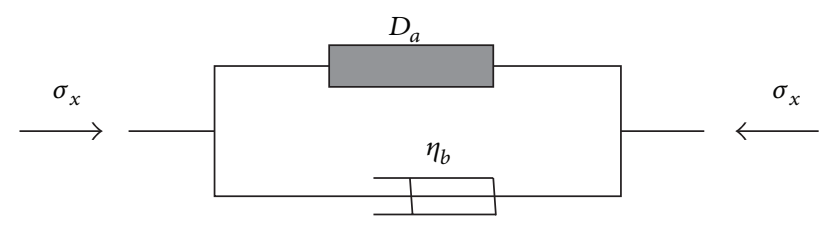

FIgURE 1: The viscoelastic damage model of shale.

the effective stress on a microscale to increase. At the same time, due to the increasing effective stress, more defects are produced. Thus, the strength of the whole rock is reduced. Under the condition of continuous load, the changes of the damage in mechanical properties can be seen as a successive process approximately. In order to analyze the process conveniently, the assumptions are shown as follows [9].

(a) Ignore the impact of inertial effect on constitutive relation within strain rate's scope of $10 \sim 10^{2} / \mathrm{s}$.

(b) Shale is treated as isotropic viscoelastic on a macroscale. The viscoelastic damage model of shale is shown in Figure 1. In addition, the damage attributes are isotropic as well.

(c) Shale obeys Hooke's law and the unit of shale is viscoelasticity before damage.

(d) In a resistivity model, when it is under static load conditions, the viscous body is invalid, while, under the condition of dynamic load, the viscous body plays a role in the process of impact compression.

(e) The strength of elastic body $F$ obeys the law of probability statistics.

On the basis of the above assumptions, the functions of dynamic damage constitutive have been built and, in the functions, the strength of shale obeys Weibull distribution, and the probability density function is given by

$$
P(F)=\frac{m}{F_{0}} *\left(\frac{F}{F_{0}}\right)^{m-1} \exp \left[-\left(\frac{F}{F_{0}}\right)^{m}\right],
$$

where $P(F)$ is the distribution function of shale's strength, that is, the failure probabilities of shale when the strength of the rock is $F$; $F$ is distribution variable; $F_{0}$ and $m$ are the parameters of Weibull distribution, where $F_{0}$ represents the macroscopic average strength of rock and $m$ reflects the brittleness of rock. Generally speaking, both of them reflect the mechanical properties of rock material.

2.1. The Damage Evolution Equation. The damage of shale is caused by the rising number of internal holes under the action of load. The accumulation of the damage forms the fracture damage of materials. Shale's breakage that occurs may be regarded as a destruction of microunit. At the same time, the breakage causes the effective area which can bear the load to decrease. On a certain level of load, the failure area is $A_{p}$, and the damage variable is defined as the ratio of failure area $A_{p}$ and total area $A$; that is, $D=A_{p} / A$; at any interval
$[F, F+d F]$, the failure area is $A P(F) d F$, and when the load is added to $F$, the failure area can be shown as

$$
A_{p}(F)=\int_{0}^{F} A P(F) d F=A\left\{1-\exp \left[-\left(\frac{F}{F_{0}}\right)^{m}\right]\right\} .
$$

The damage evolution equation is

$$
D=\frac{A_{p}(F)}{A}=1-\exp \left[-\left(\frac{F}{F_{0}}\right)^{m}\right]
$$

2.2. The Dynamic Damage Constitutive Equation. In 1984, the strain equivalence principle was put forward by the French famous scholar Lemaitre [10], which was defined as the strain occurring to damage materials under the stress that was equivalent to the materials in the same condition under the effective stress. And shale's basic formula of damage constitutive relation can be established by

$$
\left\{\sigma^{*}\right\}=\frac{\{\sigma\}}{(1-D)}=\frac{[C]\{\varepsilon\}}{(1-D)},
$$

where $\{\sigma\}$ is the nominal stress vector, $\left\{\sigma^{*}\right\}$ is the effective stress vector, $[C]$ is shale's elastic matrix, $\{\varepsilon\}$ is the strain matrix, and $D$ is the damage variable. The effective stress is based on the assumption that stress cannot transfer through the damage, but, by the previous research results, it can be known that damages like cracks, holes, and joints can transfer part of the stress in the process of compression. Based on the above reasons, the damage correction factor $q$ which ranges from 0 to 1 is introduced in the constitutive model. Therefore, formula (4) can be modified as

$$
\left\{\sigma^{*}\right\}=\frac{\{\sigma\}}{(1-q D)}=\frac{[C]\{\varepsilon\}}{(1-q D)} .
$$

In this paper, shale's damage criterion $f(\sigma)$ is set as random distribution variable and is given by

$$
F=f(\sigma)
$$

Because the Drucker-Prager yield criterion has the characteristics of simple form and suitability for rock medium [11], it is determined to express shale's yield strength, which is

$$
F^{*}=f\left(\sigma^{*}\right)=\alpha I_{1}^{*}+\sqrt{J_{2}^{*}}
$$

where $\alpha$ is the function of shale's friction angle and cohesion, and the related equation follows as $\alpha=\sin \varphi / \sqrt{9+3(\sin \varphi)^{2}}$, with the value of $\alpha$ ranging from 0 to $\sqrt{3} ; I_{1}^{*}$ is the first stress invariant; $J_{2}^{*}$ is the second deviatoric stress invariant; and the formulas, respectively, are expressed as follows:

$$
\begin{aligned}
& I_{1}^{*}=\sigma_{1}^{*}+\sigma_{2}^{*}+\sigma_{3}^{*}, \\
& J_{2}^{*}=\frac{1}{6}\left[\left(\sigma_{1}^{*}-\sigma_{2}^{*}\right)^{2}+\left(\sigma_{2}^{*}-\sigma_{3}^{*}\right)^{2}+\left(\sigma_{3}^{*}-\sigma_{1}^{*}\right)^{2}\right] .
\end{aligned}
$$


By Hooke's law, it can be gotten that

$$
\begin{aligned}
\varepsilon & =\frac{\sigma_{1}^{*}}{E}, \\
\sigma_{1}^{*} & =\frac{\sigma_{1}}{1-q D} .
\end{aligned}
$$

Because of the uniaxial compression in this paper, it can be known that

$$
\sigma_{2}^{*}=\sigma_{3}^{*}=0
$$

So, by formulas (8)-(10), it can be obtained that

$$
\begin{gathered}
I_{1}^{*}=\sigma_{1}^{*}, \\
\sqrt{J_{2}^{*}}=\frac{E \varepsilon}{\sqrt{3}} .
\end{gathered}
$$

Assuming that the shale is under dynamic loads in the $x$ direction, the viscosity of the body in $x$-direction will play a role. And it can be seen that the strain of elastic body $\varepsilon_{1}$ is equal to the viscous body $\varepsilon_{2}$, and the sum of the two is the apparent stress value in $x$-direction, which is expressed by the following formula:

$$
\begin{aligned}
\varepsilon_{1} & =\varepsilon_{2}=\varepsilon_{x}, \\
\sigma_{1}+\sigma_{2} & =\sigma_{x} .
\end{aligned}
$$

So, the constitutive model of elastomeric is

$$
\begin{aligned}
\sigma_{1}= & E \varepsilon_{x}(1-q D)+\mu\left(\sigma_{y}+\sigma_{z}\right) \\
= & E \varepsilon_{x}\left\{1-q\left\{1-\exp \left[-\left(\frac{F}{F_{0}}\right)^{m}\right]\right\}\right\} \\
& +\mu\left(\sigma_{y}+\sigma_{z}\right) .
\end{aligned}
$$

For viscous part, because there is no damage characteristic, its constitutive relation is

$$
\sigma_{2}=\eta \frac{d \varepsilon_{2}}{d t}=\eta \frac{d \varepsilon_{x}}{d t}
$$

Because of the uniaxial compression, there is

$$
\sigma_{y}=\sigma_{z}=0
$$

Substitute formulas (13), (14), and (15) into formula (12) and the constitutive relation of shale parallel body is obtained as

$$
\begin{aligned}
\sigma_{x}= & E \varepsilon_{x}\left\{1-q\left\{1-\exp \left[-\left(\frac{\alpha I_{1}+J_{2}^{1 / 2}}{F_{0}}\right)^{m}\right]\right\}\right\} \\
& +\eta \frac{d \varepsilon_{x}}{d t}
\end{aligned}
$$

Formula (16) is shale's dynamic damage constitutive model based on Weibull distribution.
2.3. Determination of Parameters in Constitutive Model. In the shale's dynamic damage constitutive model, six parameters, $E, \alpha, \eta, m, F_{0}$, and $q$, need to be determined [12]. $E$ can be measured by the slope of the initial rise stage of the shale's stress-strain curve; the value of $\alpha$ ranges from 0 to $\sqrt{3}$; and that of $\eta$ ranges from 0.1 to 0.5 ; the strain and strain rate are measured by experiments, while $m, F_{0}$, and $q$ can be determined by fitting the shale's stress-strain curves of impact shock experiment.

For the experiment of uniaxial compression of shale, the axial stress is set as $\sigma$ and the corresponding strain is set as $\varepsilon$, and then the shale's dynamic statistical damage constitutive model can be established by

$$
\sigma=E \varepsilon\left\{q \exp \left[-\left(\frac{\alpha I_{1}+J_{2}^{1 / 2}}{F_{0}}\right)^{m}\right]+1-q\right\}+\eta \frac{d \varepsilon}{d t}
$$

The formula above can be reduced to

$$
\frac{\sigma+\eta(d \varepsilon / d t)}{E \varepsilon}+q-1=q \exp \left[-\left(\frac{\alpha I_{1}+J_{2}^{1 / 2}}{F_{0}}\right)^{m}\right] .
$$

Taking the logarithm on both sides of formula (18), there is

$$
\begin{aligned}
& \ln \left[\frac{\sigma+\eta(d \varepsilon / d t)}{E \varepsilon}+q-1\right]=\ln q-\left(\frac{\alpha I_{1}+J_{2}^{1 / 2}}{F_{0}}\right)^{m}, \\
& -\ln \left[\frac{\sigma+\eta(d \varepsilon / d t)}{E \varepsilon}+q-1\right]+\ln q \\
& =\left(\frac{\alpha I_{1}+J_{2}^{1 / 2}}{F_{0}}\right)^{m}=\frac{1}{F_{0}^{m}}\left(\alpha I_{1}+J_{2}^{1 / 2}\right)^{m}=a k^{m} .
\end{aligned}
$$

Taking the logarithm on both sides of formula (20), there is

$$
\begin{aligned}
& \ln \left\{-\ln \left[\frac{\sigma+\eta(d \varepsilon / d t)}{E \varepsilon}+q-1\right]+\ln q\right\} \\
& \quad=\ln a+m \ln k
\end{aligned}
$$

and set

$$
\begin{aligned}
& y=\ln \left\{-\ln \left[\frac{\sigma+\eta(d \varepsilon / d t)}{E \varepsilon}+q-1\right]+\ln q\right\}, \\
& x=\ln k \\
& b=\ln a .
\end{aligned}
$$

Equation (21) can be simplified as

$$
y=m x+b
$$

By linear regression, $m$ and $b$ can be obtained, and then according to

$$
F_{0}=\exp \left(-\frac{b}{m}\right)
$$

$F_{0}$ is obtained. 
TABLE 1: Parameters of the shale's dynamic statistical damage constitutive model.

\begin{tabular}{lccccc}
\hline Specimen number & Strain rate $\left(\dot{\varepsilon} / \mathrm{s}^{-1}\right)$ & $F_{0} /(\mathrm{MPa})$ & $m$ & $q$ & Correlation coefficient \\
\hline 1 & 173.2 & 352.426 & 3.0014 & 0.9854 & 0.9274 \\
3 & 86.4 & 147.604 & 2.5506 & 0.9945 & 0.9894 \\
4 & 35.2 & 85.218 & 5.0683 & 1 & 0.9864 \\
5 & 205.6 & 482.463 & 3.7822 & 0.9831 & 0.9069 \\
6 & 164.8 & 286.654 & 5.7884 & 0.9876 & 0.8825 \\
7 & 45.7 & 93.391 & 3.8625 & 0.9987 & 0.9834 \\
\hline
\end{tabular}

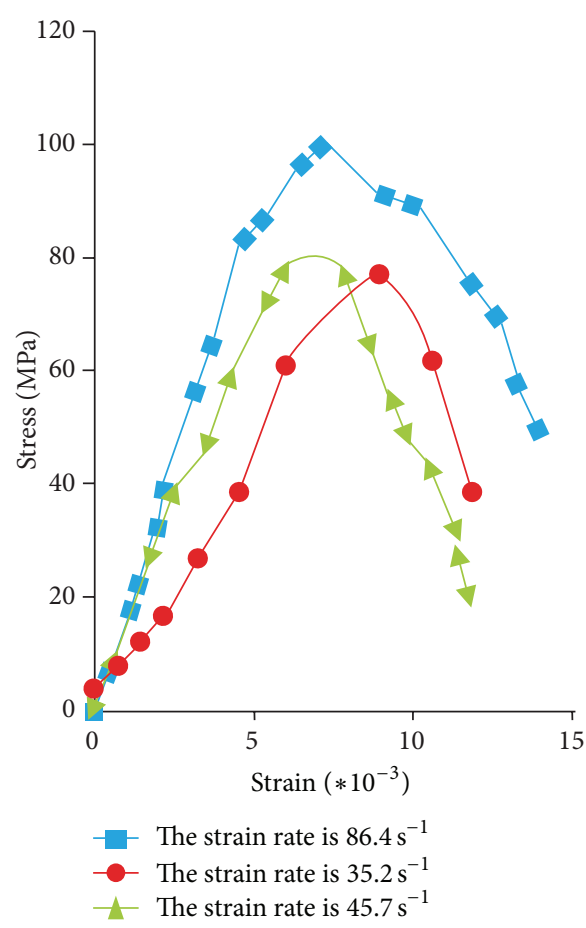

(a)

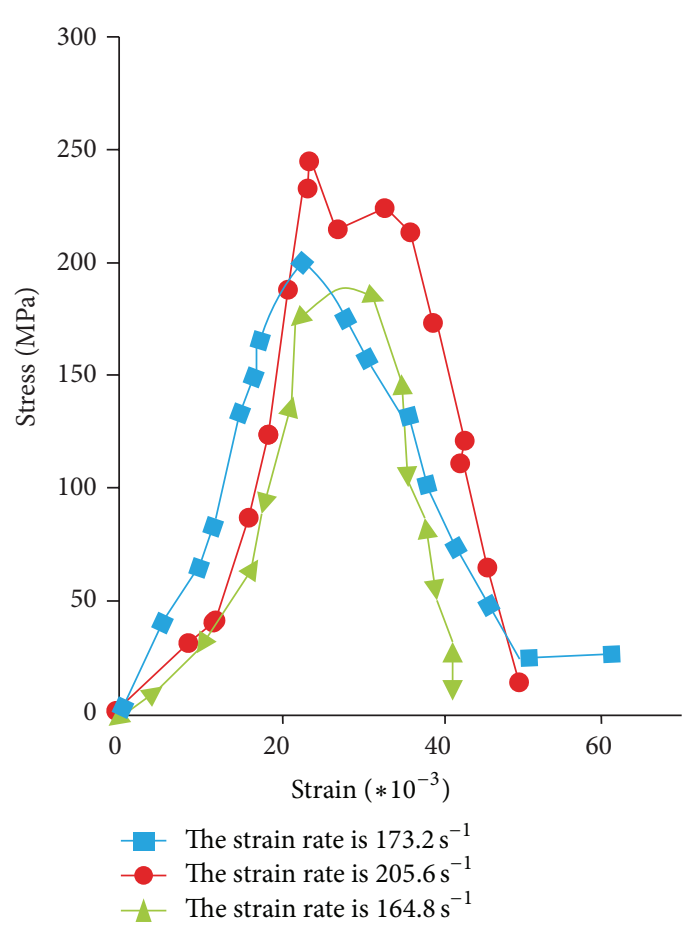

(b)

FIGURE 2: The stress-strain curves under different strain rates.

The solving process above is to obtain $m$ and $F_{0}$ by using curve fitting method. Then, substitute the values into formula (3) and formula (16), and the shale's damage evolution equation and statistical damage constitutive model under impact load can be obtained.

Through the SHPB impact test for shale, the obtained test results of the shale's stress-strain curve are shown in Figure 2.

Using the above method, in the shale's dynamic statistical damage constitutive model, parameters $F_{0}$ and $m$ under different strain rates can be obtained, as shown in Table 1.

\subsection{The Correction of Constitutive Model}

2.4.1. The Effect of Weibull Distribution Parameters $F_{0}$ and $m$ on the Constitutive Model. In order to study the effect of Weibull distribution parameters $F_{0}$ and $m$ on constitutive model, a univariate method is selected [13]. First of all, fix $m$, and study the changes in the constitutive curve when $F_{0}$ changes. Then, fix $F_{0}$, and study the changes of constitutive curve accompanying the value of $m$. According to the experimental results of $3 \#$ test specimen the analysis results are shown in Figures 3 and 4.

From Figures 3 and 4, the following rules can be concluded.

(1) The shale's stress-strain curve peak increases with the increase of distribution parameters $F_{0}$ and $m$.

(2) The effect of distribution parameters $F_{0}$ and $m$ on the constitutive curve after the peak is larger than before the peak.

\subsubsection{The Effect of Damage Correction Factor q on Constitutive} Model Curve. According to the experimental result of $7 \#$ test specimen, the effect of damage correction factor $q$ on constitutive model curve is studied and the result is shown in Figure 5.

Figure 5 shows that, with the decrease of $q$, the shale's peak strength increases, the ductility increases after the peak, and the residual strength increases. 


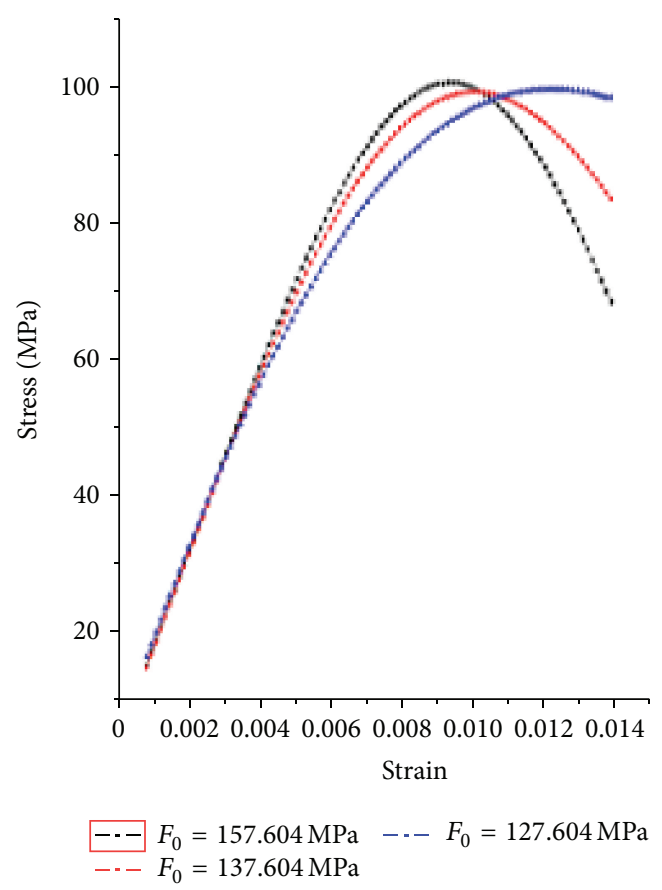

FIgURE 3: The effect of parameter $F_{0}$ on constitutive curve.

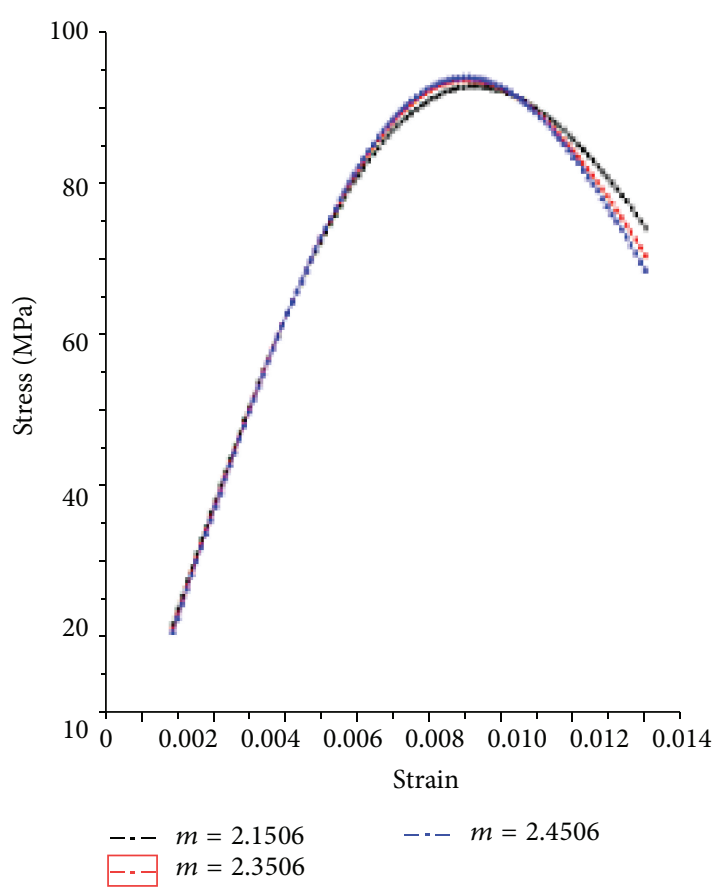

Figure 4: The effect of parameter $m$ on constitutive curve.

2.4.3. The Correction Process of the Constitutive Model. According to the Weibull distribution parameters $F_{0}$ and $m$ obtained above, the strain rate is set as horizontal axis, and $F_{0}$ and $m$ areas are set as vertical axis, respectively. The curves changing with the strain rate are obtained, and the results are shown in Figure 6.

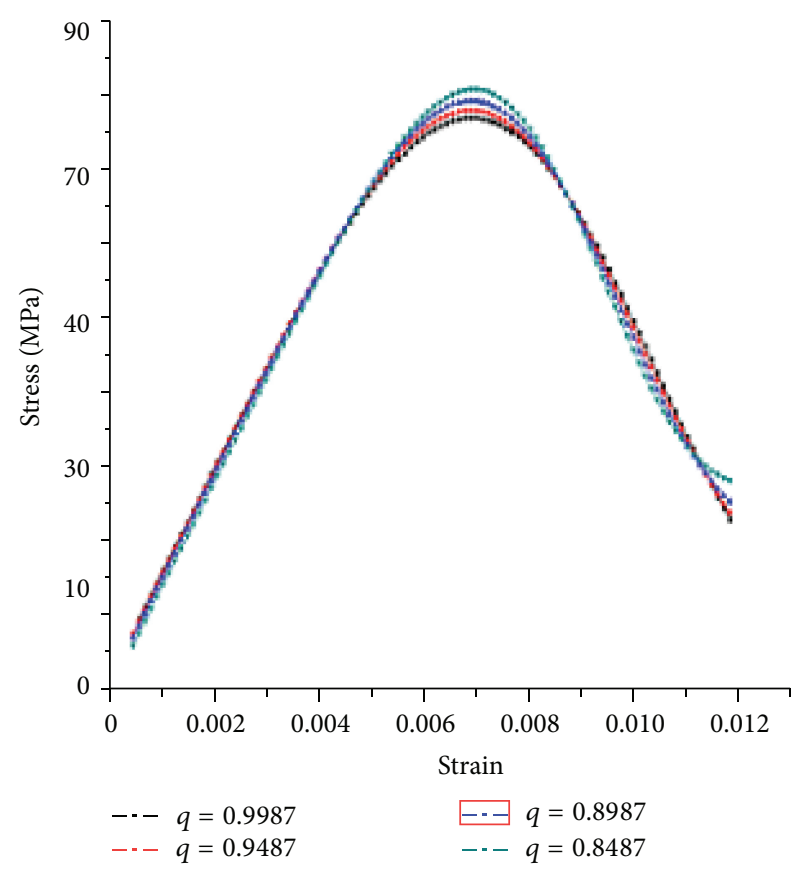

FIGURE 5: The effect of damage correction factor $q$ on constitutive curve.

By fitting the plot, the relationship between $F_{0}$ and $m$ with strain rate is established and expressed by

$$
\begin{aligned}
F_{0} & =2.1531 \dot{\varepsilon}-13.8088, \\
m & =7.6149-0.0864 \dot{\varepsilon}+3.3410 E(-4)(\dot{\varepsilon})^{2} .
\end{aligned}
$$




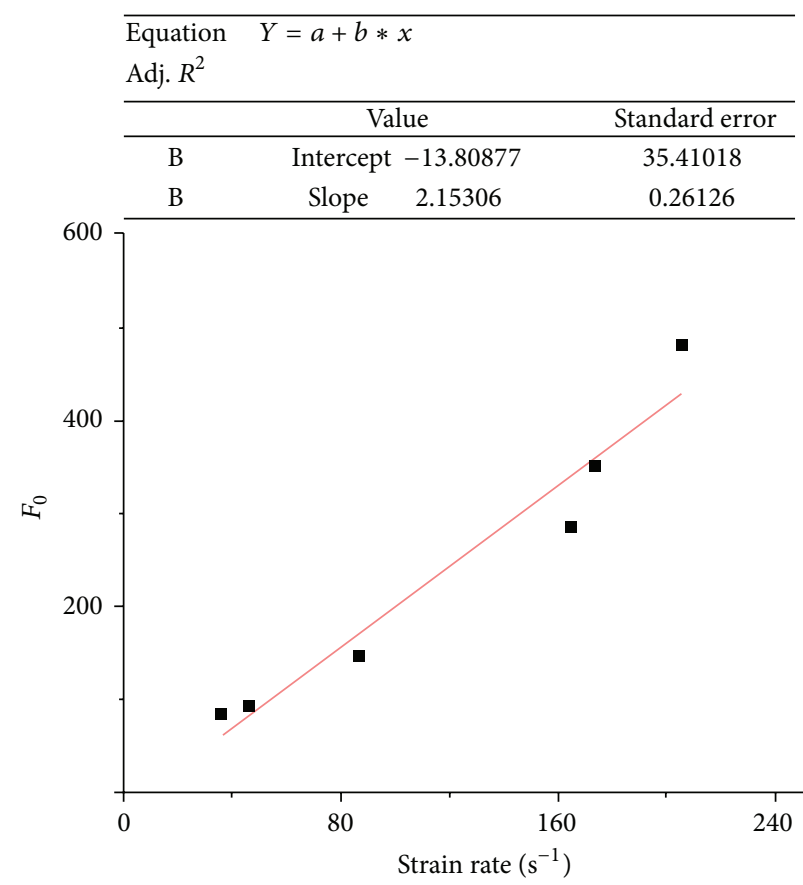

$F_{0}$

Linear fit of $F_{0}$

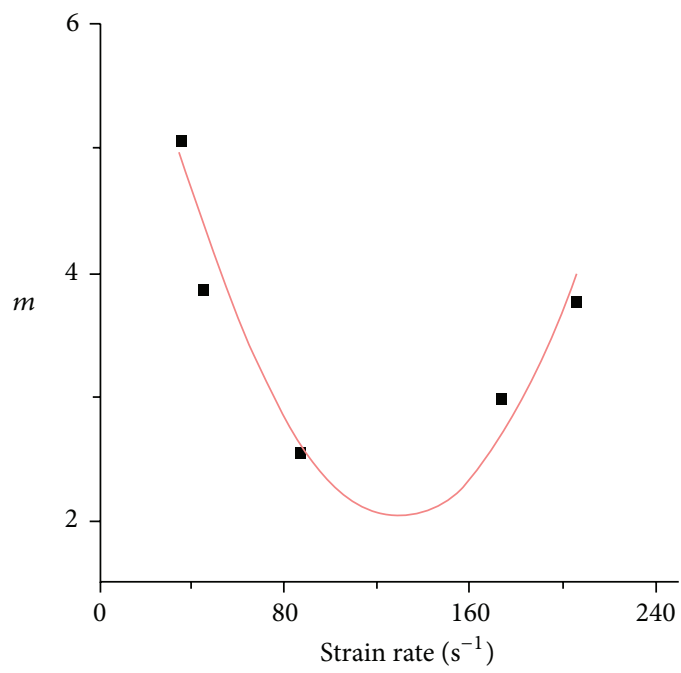

$m$

Polynomial fit of $m$

FIGURE 6: The relationship between model parameters $F_{0}$ and $m$ with strain rate $\dot{\varepsilon}$.

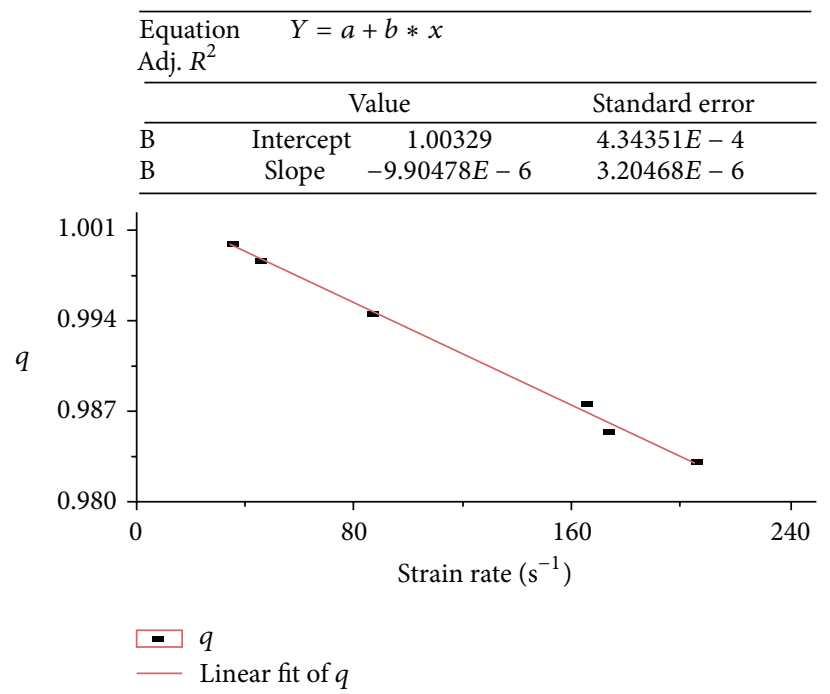

FIGURE 7: Relationship between damage correction factor $q$ and strain rate $\dot{\varepsilon}$.

As shown in Figure 7, the relationship between the damage correction factor $q$ and strain rate $\dot{\varepsilon}$ turns out to be obviously linear and the equation of linear fitting is

$$
q=1.0033-9.9048 E(-5) \dot{\varepsilon} .
$$

Substitute formulas (25) and (26) into the constitutive equation (16) and the shale's dynamic statistical damage constitutive model of specific solutions can be obtained.
2.5. Verification of Constitutive Model. The parameters of the statistical constitutive model can be solved by fitting the stress-strain curves, and then the theoretical curves can be obtained. Compared with the experimental curves, the shale's dynamic statistical damage constitutive model is verified to be correct and reasonable, and the comparison of theoretical curve and experimental curve is shown in Figure 8.

From Figure 8, it can be seen that, in this paper, the shale's dynamic damage constitutive model based on the statistical 


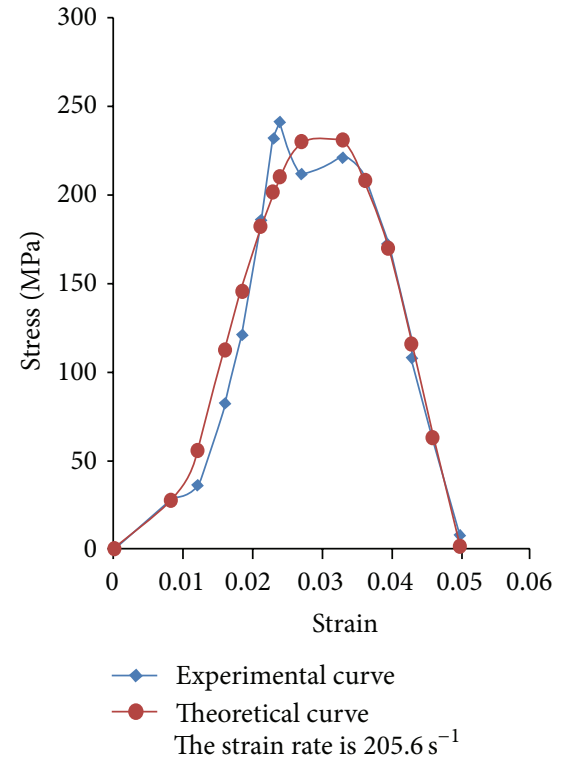

(a)

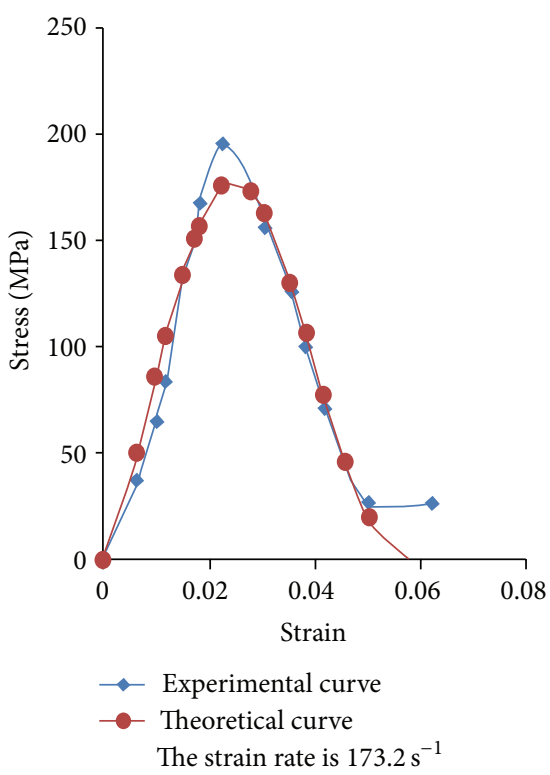

(b)

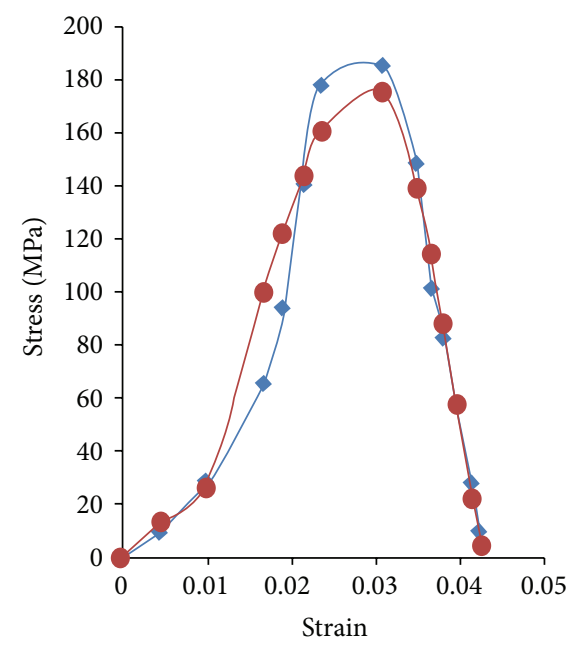

- Experimental curve

Theoretical curve The strain rate is $164.8 \mathrm{~s}^{-1}$

(c)

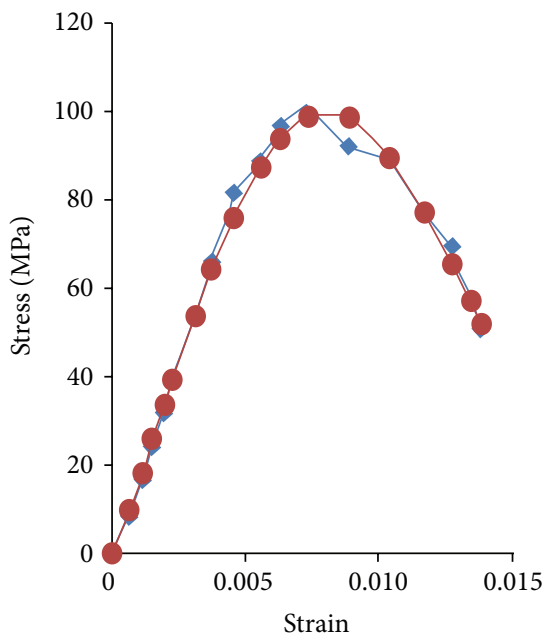

- Experimental curve

- Theoretical curve The strain rate is $86.4 \mathrm{~s}^{-1}$

(d)

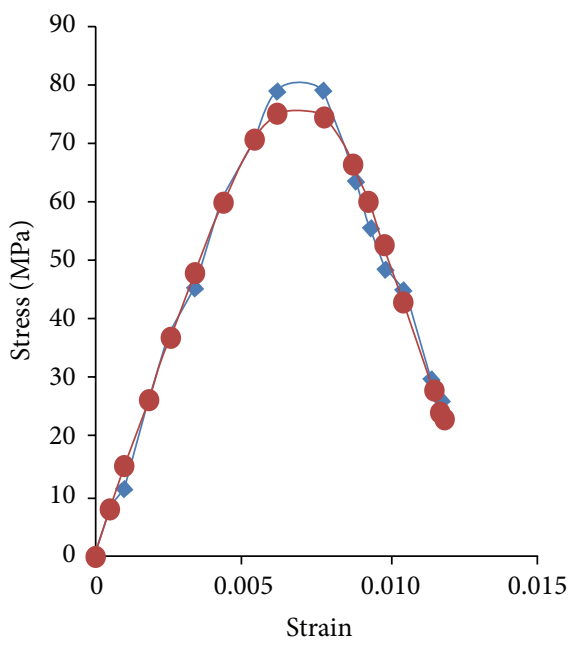

$$
\begin{aligned}
& - \text { Experimental curve } \\
& - \text { Theoretical curve } \\
& \text { The strain rate is } 45.7 \mathrm{~s}^{-1}
\end{aligned}
$$

(e)

Figure 8: Comparison of theoretical curve and experimental curve.

distribution can better reflect the shale's stress-strain relationship. Theoretical curves and experimental curves have a good alignment, which illustrates that the constitutive model established in this paper is reasonable.

\section{Conclusions}

(1) Shale's stress-strain curves will change in line with the model parameters, while the curves of the peak strength increase with the Weibull distribution parameters $F_{0}$ and $m$ increasing and decrease with the damage correction factor $q$ increasing.
(2) Parameters $F_{0}$ and $q$ of the statistical damage constitutive model and strain rate $\dot{\varepsilon}$ abide by a linear relationship, while $m$ and strain rate $\dot{\varepsilon}$ abide by the quadratic polynomial relationship.

(3) The statistical damage constitutive model is proved to be reasonable by the comparison of theoretical curves and experimental curves.

\section{Conflict of Interests}

The authors declare that there is no conflict of interests regarding the publication of this paper. 


\section{Acknowledgment}

The research is financially supported by the National Science Foundation of China (Grant no. 51174170).

\section{References}

[1] U. S. Lindholm, L. M. Yeakley, and A. Nagy, "The dynamic strength and fracture properties of dresser basalt," International Journal of Rock Mechanics and Mining Sciences \& Geomechanics Abstracts, vol. 11, no. 5, pp. 181-191, 1974.

[2] K. Sigenori, S. Azuhiko, and K. Minoru, "On the mechanical behavior of rocks under impulsive loading," Bulletin of the Faculty of Engineering Hokkaido University, vol. 83, pp. 51-62, 1977.

[3] Y. Yu, Rock Dynamics, University of Science and Technology Beijing Press, Beijing, China, 1994.

[4] D. Krajcinovic and M. A. G. Silva, "Statistical aspects of the continuous damage theory," International Journal of Solids and Structures, vol. 18, no. 7, pp. 551-562, 1982.

[5] J. W. Dougill, J. C. Lau, and N. J. Burt, "Toward a theoretical model for progressive failure and softening in rock, concrete and similar material," in Proceedings of the ASCE-EMD Specialty Conference on Mechanics in Engineering, pp. 335-355, Waterloo, Canada, May 1976.

[6] Y. Chen and S. Feng, "Lagrangian analysis method and constitutive relation of cement stone on intense dynamic loading," Chinese Academy of Railway Sciences, vol. 17, no. 3, pp. 1-11, 1996.

[7] G. Wenxue, Y. Jun, and H. Fenglei, "The constitutive relation of rock under strong impact loading," Journal of Beijing Institute of Technology, vol. 20, no. 2, pp. 165-170, 2000.

[8] L. Shang and J.-G. Ning, "Dynamic constitutive relationship of concrete subjected to shock loading," Engineering Mechanics, vol. 22, no. 2, pp. 116-120, 2005.

[9] X. Jinyu, F. Jianshe, and L. Xiaocong, Dynamic Mechanical Properties of Rock under the Condition of Confining Pressure, Northwestern Polytechnic University Press, 2012.

[10] J. A. Lemaitre, "How to use damage mechanics," Nuclear Engineering and Design, vol. 80, no. 2, pp. 233-245, 1984.

[11] J. Liu, L. Lin, R. Song, and J. Zhao, "A pore scale modeling of fluid flow in porous medium based on navier-stokes equation," Disaster Advances, vol. 6, supplement 1, pp. 5-11, 2013.

[12] Q. He, L. Dianshu, and W. Shuren, "Experimental study of rock's constitutive relation on high strain ration," Blasting, vol. 13, no. 2, pp. 1-7, 1996.

[13] W. Cao, M. Zhao, and C. Liu, "Study on the model and its modifying method for rock softening and damage based on Weibull random distribution," Chinese Journal of Rock Mechanics and Engineering, vol. 23, no. 19, pp. 3226-3231, 2004. 

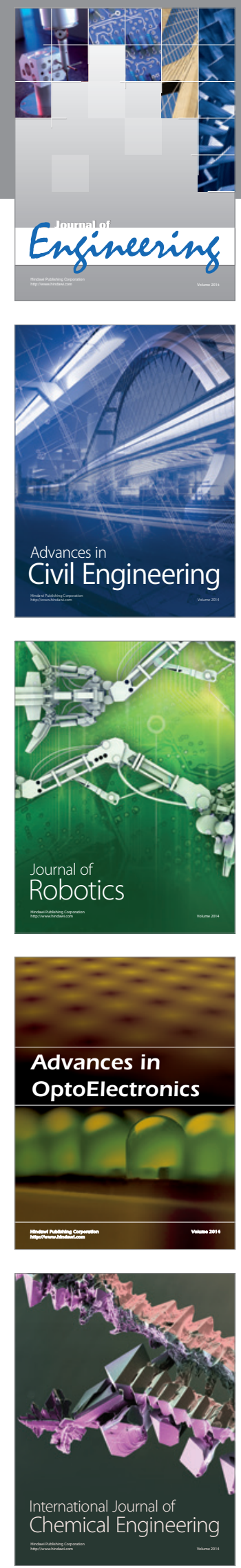

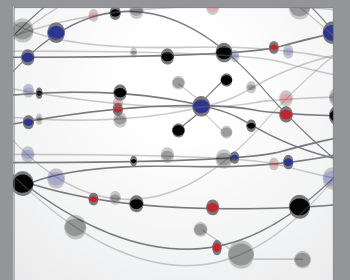

The Scientific World Journal
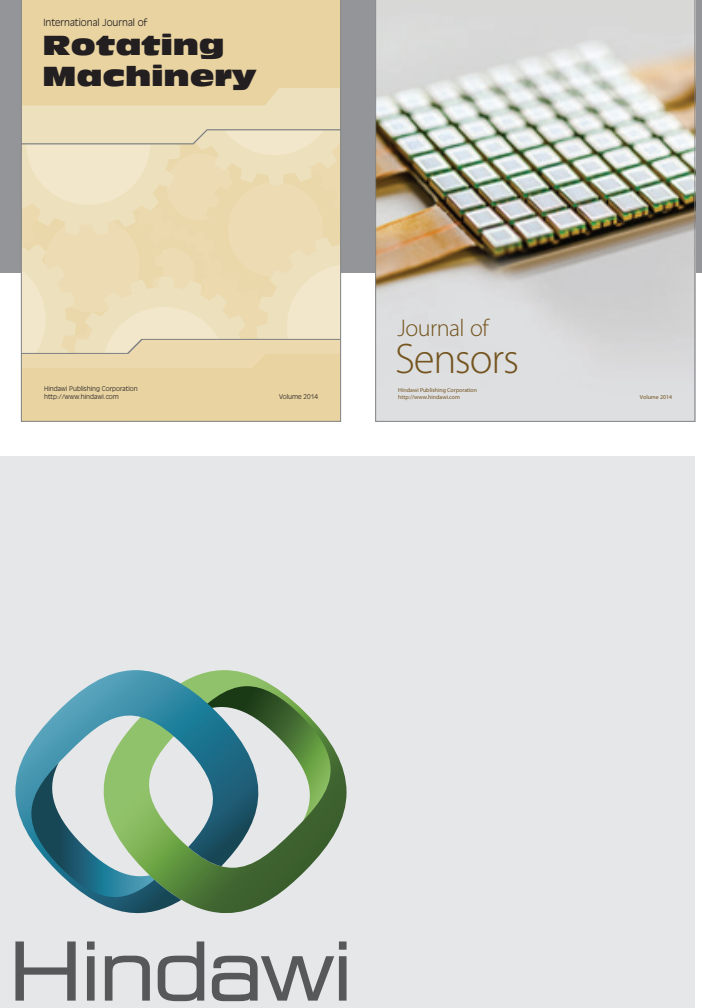

Submit your manuscripts at http://www.hindawi.com
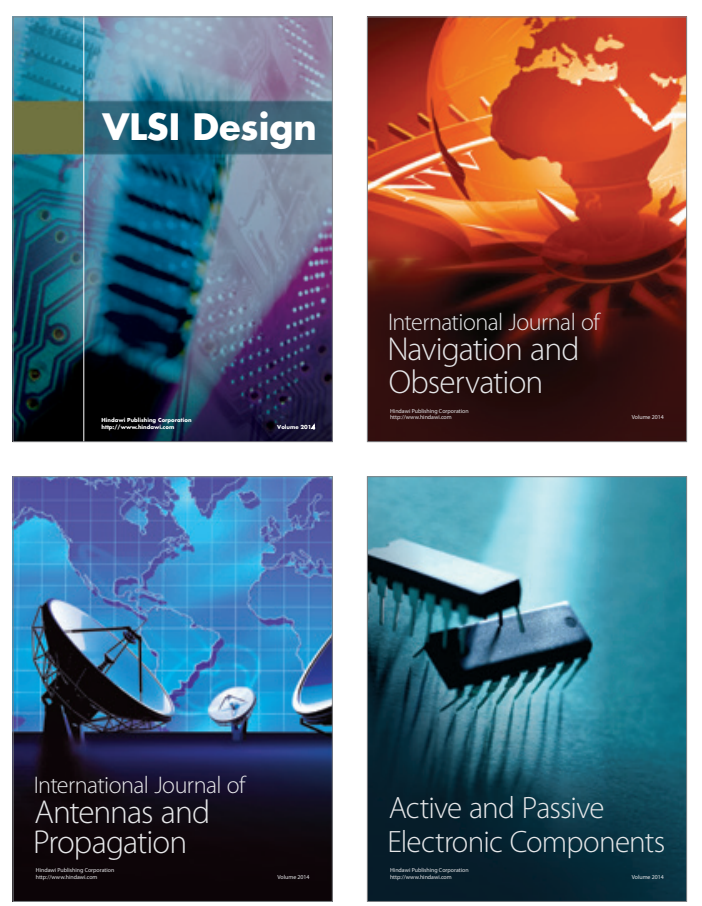
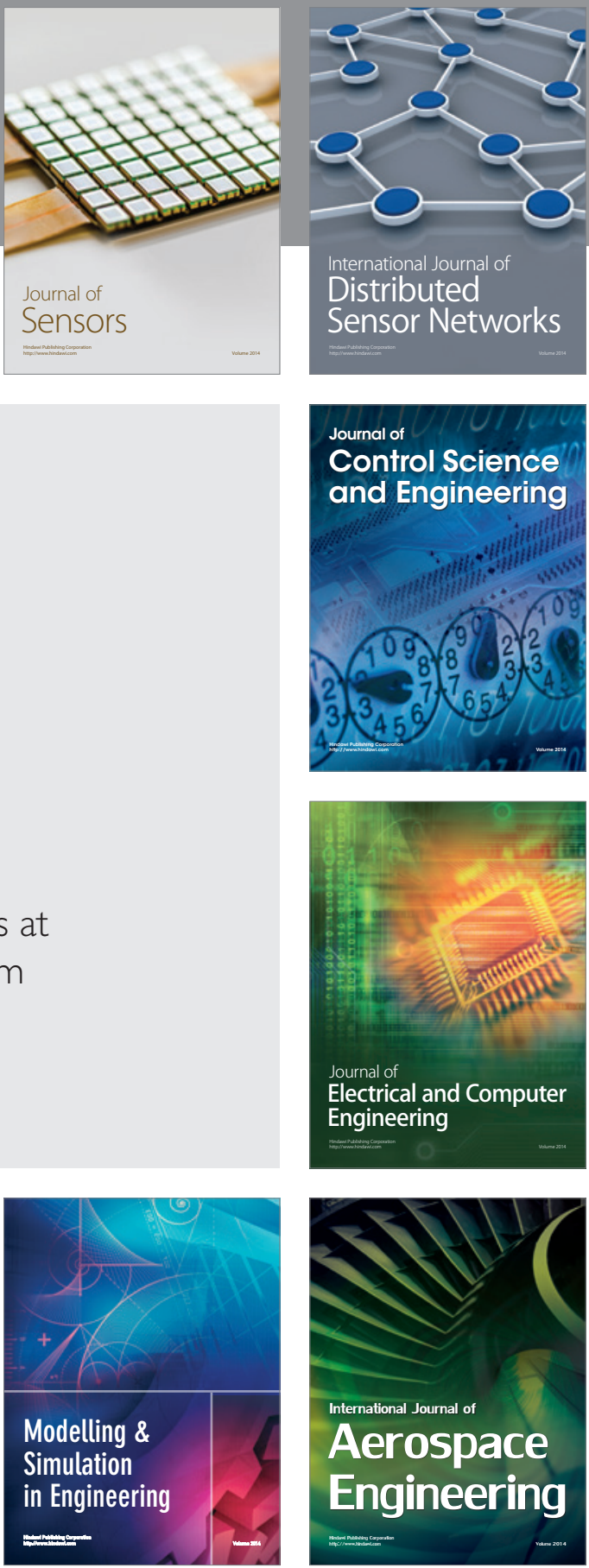

Journal of

Control Science

and Engineering
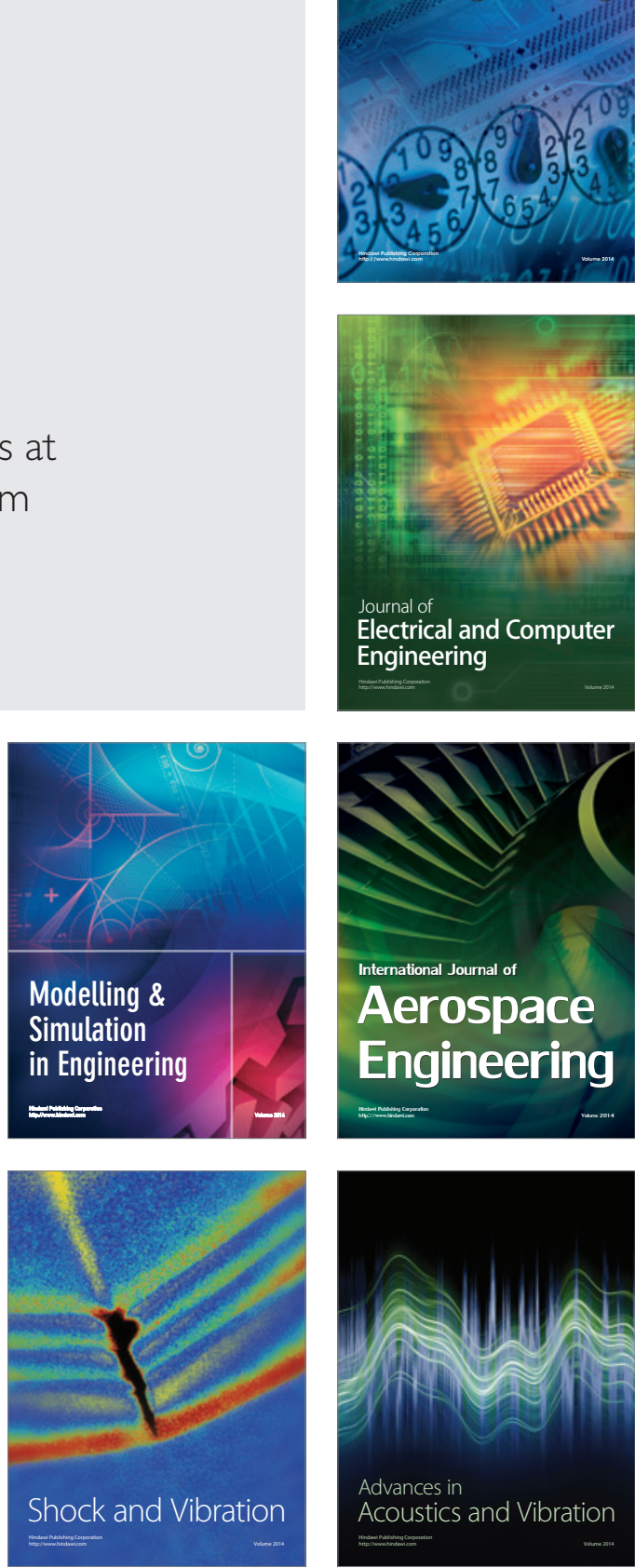